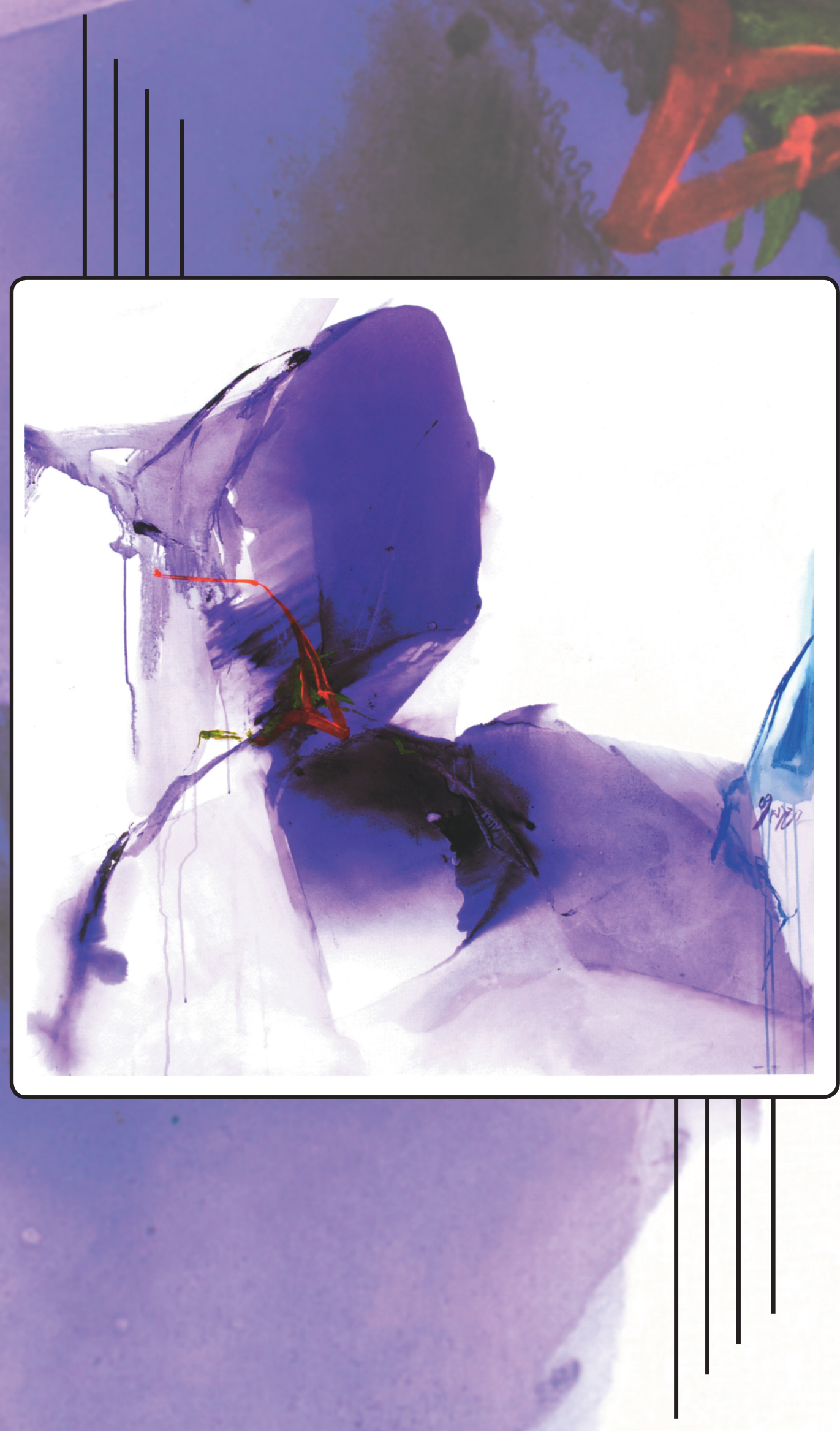


Hommage aux 12 apotres

Óleo sobre Lienzo

$120 \times 120 \mathrm{cms}$

2009

Colección Privada Universidad Santo Tomás 


\title{
INSTRUMENTOS DE RESPONSABILIDAD SOCIAL EMPRESARIAL VISTOS POR ORGANIZACIONES NO GUBERNAMENTALES, ONG
}

María G. Daza

\section{Resumen}

La investigación ${ }^{2}$ se fundamenta en revisión documental y una lectura analítica de los archivos, publicaciones y declaraciones de cuatro Organizaciones No Gubernamentales (ONG) quienes expresan su posición sobre la efectividad de algunos de los Códigos de Conducta más conocidos.

El estudio clasifica inicialmente los Códigos de Conducta en dos grupos: privados y públicos, con el fin de identificar la procedencia de los códigos; los privados son la herramienta más usada, por presentar algunas ventajas, por ser una iniciativa propia de la empresa para comprometerse y formalizar un Código de Conducta interno. Los públicos tienen cobertura internacional, consisten en un contrato entre sujetos del Derecho Internacional, por esta razón, tienen un grado de competencia jurídica, además de contar con el factor supranacional. En la actualidad se debate sobre la funcionalidad y el objetivo de los Códigos de Conducta.

\section{Palabras Clave}

Códigos de Conducta, Responsabilidad Social Empresarial (RSE) y Derechos Humanos.

\begin{abstract}
This research is based on a bibliographical review of publications and other public documents where Non-Government Organizations (NGO), are given out a concept and their position about how effective some of the Codes of Conduct are.

In the study intruduces with a classification of the initiatives, between private or public to identify where the codes are coming from. Thereby, the Private Codes of Conduct are one of the most useful tools because of its advantages, such as being the enterprise own initiatives to compromise and formalize an internal Code of Conduct. The Public Codes have an international covering that consist of a contract among subjects of the International Law, the States, for this reason, all the States have a juridical competence degree; furthermore, they rely on a supranational factor. Nowadays, there is a debate about the Codes of Conduct utility and objectives.
\end{abstract}

\section{Key Word}

Code of Conduct, Corporate Social Responsability, Human Rights.

1 Maestría en Estudios Europeos. Docente- Investigadora Economía Social Facultad de Economía Universidad Santo Tomás Bucaramanga.

2 Este artículo es parte de la investigación: Organizaciones No Gubernamentales y su posición sobre los Códigos de Conducta para Empresas Multinacionales, desarrollada para optar por el título de Maestría en Estudios Europeos de la Universidad de Hamburgo. 


\section{Introducción}

En los últimos años algunas multinacionales se han visto envueltas en demandas, sobre todo referentes a la violación de derechos humanos, por la explotación laboral hacia sus empleadas y empleados y el maltrato del medio ambiente; por ejemplo, las maquilas en zonas de libre comercio en Centroamérica y en Asia, o en denuncias por violación a los derechos humanos de sindicalistas en el contexto de conflicto armado en Colombia o casos de apoyo financiero a grupos políticos cuestionados por supuesto apoyo en masacres en África, con el objeto de poder tener acceso a la materia prima (Strutynski \& Platiel, 2005).

Actualmente estos casos ocurren no sólo en países del hemisferio sur, sino en todos los países del mundo, donde la capacidad del Estado para la aplicación y el control de las leyes no funciona efectivamente; estos casos ocurren también en países donde, cada vez, el ser humano se convierte en instrumento para lograr objetivos y no objetivo de las políticas.

Las empresas han ganado poder en el mundo globalizado; ya que influyen en muchos ámbitos económicos y políticos. Algunos factores que han consolidado esta situación son la privatización de los bienes públicos, el avance tecnológico y, por supuesto, la internacionalización de la economía (Fonari, 2004). De igual manera las empresas influyen en la sociedad, como en su personal, en otras compañías filiales, intermediarios, proveedores y consumidores. En este sentido las ventajas comparativas de la cadena productiva global son igualmente una razón para la creciente expansión de las empresas en el mundo. Lo cual es una ventaja que permite que las empresas produzcan en el lugar del mundo donde las condiciones son óptimas $\mathrm{y}$ ventajosas (mayores ganancias).

Dentro de las condiciones y factores que atraen a inversionistas, en un contexto de economía de mercado, cabe mencionar que los inversionistas prefieren desarrollar los procesos de producción en países con menores costos salariales, en países con mercados abiertos, con programas de privatización, que apliquen tratados internacionales, además de tener en cuenta la disponibilidad de recursos de algunos países (Hamm, 2004). En este orden de ideas, para los gobiernos puede resultar atractivo atraer empresas que inviertan en el país y generen puestos de trabajo. Pero sin las adecuadas normatividades sociales y de medio ambiente, las empresas pueden aprovechar la situación e incurrir en actividades económicas y políticas irresponsables. La investigación se trabajó mediante el análisis de la efectividad de algunos Códigos de Conducta, como instrumento de la Responsabilidad Social Empresarial, y si estos realmente pueden aportar al mejoramiento del comportamiento de las empresas con respecto a los derechos humanos.

El concepto de Responsabilidad Social Empresarial, se fortalece en los últimos años como una herramienta que usan los agentes económicos, en este caso las 
empresas, para mejorar la imagen. Este no es un término del siglo XXI, en los años 60, Milton Friedmann lo menciona en su obra "Capitalismo y Libertad", y afirma en un artículo de la revista The New York Times: "la responsabilidad social es para incrementar las ganancias (...) pues son las personas, quienes pueden tener responsabilidades, las empresas tienen responsabilidades artificiales" (1970). El concepto toma fuerza en la actualidad cuando las empresas dedican esfuerzos para procurar dentro de su actividad económica el respecto, la defensa y protección de los derechos humanos. Hasta convertirse, hoy día, en un debate académico que relaciona la responsabilidad social empresarial con la ética empresarial, lo que lleva a discusiones sobre la concepción de la empresa como una institución con responsabilidades y deberes públicos (Lozano y Siurana, 2000).

La RSE está dirigida a proteger los Derechos Humanos, pero más a evitar cualquier violación de ellos. Al mencionar los derechos humanos se incluyen todas las generaciones, esto quiere decir que la primera generación la componen los derechos civiles y políticos, entre ellos, los derechos fundamentales, derecho a la vida, la libertad, la igualdad y otros; la segunda generación la constituyen los derechos colectivos, entre ellos, los derechos sociales, económicos y culturales; y la tercera generación son los derechos de los pueblos entre ellos al ambiente sano, a la paz, al desarrollo. Es por esta razón que no se considera necesario mencionar los estándares sociales y los estándares ambientales de forma separada, éstos están incluidos dentro del concepto generacional de los derechos humanos.

\section{Códigos de Conducta}

Los Códigos de Conducta para empresas multinacionales son reglas, normas o pautas, que reglamentan el comportamiento de las empresas en sus actividades económicas, son instrumentos prácticos que usan las empresas para hacer programas de Responsabilidad Social Empresarial (RSE).

"Los Códigos de Conducta son directrices escritas, que sirven como fundamento para el comportamiento de las empresas multinacionales hacia sus trabajadores, empresas filiales, proveedores, estamentos del gobierno y el medio ambiente" (Greven, 2004, p.151).

Los códigos deben tener como objetivo final el reconocimiento de las personas como tales en la actividad económica, de tal forma que ellas sean objetivo final de las empresas y no los medios para lograr fines particulares. Estos instrumentos, los códigos, sirven no sólo a las empresas multinacionales, sino también a cualquier institución para iniciar un programa tanto interno, como externo de responsabilidad social, actualmente existe una gran variedad de instrumentos que ayudan de forma práctica a introducir este concepto dentro de la actividad institucional. 
Los Códigos trabajados en este artículo son los más conocidos, usados por las empresas actualmente $\mathrm{y}$, por lo tanto, usados por las ONG con respecto a su nivel de efectividad. Se pueden clasificar de forma muy general como Códigos de Conducta Públicos y Privados. Los primeros, instrumentos de las organizaciones internacionales también llamados atrevidamente Códigos de Conducta, se pueden definir de esta forma por tener cobertura internacional y son una iniciativa que nace de un acuerdo o una institución internacional inicial conformada o propuesta por sujetos del derecho internacional (los Estados) y, por esta razón, tienen cierta competencia jurídica, además de contar con el factor supranacional, que los hace válidos en los países que hacen parte de la organización, un ejemplo es la Organización de las Naciones Unidas (ONU). Estas organizaciones internacionales están conformadas por Estados. Una organización internacional es aquella que va más allá de los límites nacionales, se constituyen por medio de Tratados y son democráticamente legítimas pues representan los intereses de los ciudadanos.

Estas organizaciones también se han involucrado en el tema de los Códigos de Conducta, son de gran importancia por los efectos que estos puedan tener por la cobertura que tienen sobre las naciones y fuera de ellas.

Los Códigos públicos de los cuales se analiza la posición de las ONG son las Normas ONU, el Pacto Mundial, la Declaración Tripartita de OIT y la Directriz OECD. De lo que se hace una ampliación más adelante. Al final se da una descripción de algunos códigos públicos que se han tenido en cuenta para este análisis.

La segunda clasificación corresponde a los Códigos de Conducta Privados, son una serie de reglas escritas, que se publican dentro de las empresas. En ellos se resume las responsabilidades que la empresa desea fortalecer y cómo debe ser el comportamiento de los empleados, empleadores y otros dentro de las firmas, buscan defender los derechos humanos; no tienen ningún significado jurídico, son un compendio de estándares sociales, de medio ambiente y derechos humanos que han sido creados en primera instancia por la empresa, otros son creados por organizaciones de empresarios, según A. Wawryk (en Fryas y Pegg, 2003) los códigos se subdividen de la siguiente forma: los diseñados por las organizaciones de empresas, por ejemplo, la Cámara de Comercio Internacional ICC, quienes definen la Directriz ICC, o la Organización Internacional de Estandarización (ISO) quienes desarrollan la Pauta de Responsabilidad (Responsability Guideline).

Otro grupo, son los códigos propuestos por algunas ONG, por ejemplo, el Estándar de Contabilidad Social 8000 (SA 8000). Y, finalmente, los códigos realizados por las mismas empresas: códigos privados de empresas. Ejemplo de ellos es el Código de Conducta de Adidas-Salomon AG. (Cortés y Eichler, 2004) ${ }^{3}$. Considerado como uno de los más completos. Estos se consideran de forma genera 1 más adelante, junto con 
la opinión de las ONG. De manera general se describirán los códigos privados y el concepto de las ONG sobre los mismos.

El debate que surge aquí está relacionado con el derecho internacional y consiste en la legitimidad de los Códigos y si éstos en algún momento pueden reemplazar al Derecho Internacional, ya sea porque el Derecho Internacional presenta vacíos porque no aplica o porque no es respetado. Los Códigos de Conducta son considerados ejemplos de soft law "normas blandas". Para Boyle (1999) la característica más relevante del término es que no es vinculante; consiste en normas, principios, pero no reglas; es una ley que no proviene de una resolución; es decir, que los Códigos de Conducta surgen por la ausencia de normas consolidadas, puede hacer referencia a un marco de referencia temporal, necesario en una sociedad cambiante, es un derecho informal cuya falta de obligatoriedad, no impide que tengan cierta eficacia jurídica.

Los Estados son los sujetos del derecho internacional, sólo que actualmente comparten su posición con otros actores que adquieren, cada vez, mayor influencia histórica, económica y política a nivel mundial. Es necesario compartir el escenario con organizaciones no-gubernamentales, empresas multinacionales, y otras organizaciones internacionales supra-nacionales regionales. Los asuntos son de interés para la comunidad internacional, así no pueden ser tratados de manera unilateral o con acciones internas, se necesita la participación de los diferentes actores de escenario global.

La Organización de Naciones Unidas ONU es lo más cercano a un gobierno global, con respecto a las decisiones de política mundial; los gobiernos en el mundo globalizado deben atender cada vez más normas y regulaciones internacionales. Esta institución propone dos importantes códigos de comportamiento para las empresas, el primero es el Pacto Mundial, (anteriormente llamado Pacto Global) y el segundo proyecto Normas de las Naciones Unidas de Responsabilidad para Multinacionales y otras Empresas, referentes a Derechos Humanos.

\section{- $\quad$ Normas $\mathrm{ONU}^{4}$}

La Subcomisión de Promoción y Protección de los Derechos Humanos aprobó las "Normas de las Naciones Unidas sobre las Responsabilidades de las Empresas en la Esfera de los Derechos Humanos" llamadas Normas ONU, aprobadas como parte del proceso de legalización en la ONU para que una norma sea adoptada por los Estados. Posteriormente deberán ser estudiadas y aprobadas por la Comisión de Derechos Humanos donde pueden durar cinco años o más antes de aprobadas. Las normas ofrecen una panorámica sobre principios jurídicos internacionales

4 http://www.unhchr.ch/huridocda/huridoca.nsf/(Symbol)/E.CN.4.Sub.2.2003.12.Rev.2.En 
relacionados con la Declaración Universal de los Derechos Humanos, el Derecho Internacional Humanitario y Derecho Laboral Internacional, como también sobre protección del medio ambiente, del consumidor y leyes anticorrupción. Para las empresas representa un instrumento útil que mejora su desempeño en relación con los Derechos Humanos.

La creación de un grupo de trabajo compuesto por empresas, asociaciones, ONG, gremios y organismos especializados de la ONU, tuvo como objetivo primordial la cooperación y el diálogo, para conocer las opiniones, los concejos y las propuestas de estas organizaciones en su construcción. Se considera que las Normas involucran la discusión de los sectores apropiados respecto al tema. Para K. Nowrot (2003), el desarrollo de las normas representa un intento positivo de la participación de los trabajos académicos que intentan y verifican el conocimiento sobre este tema. Además, crece el círculo para la construcción de un sistema internacional jurídico con actores más relevantes que el mismo Estado. ${ }^{5}$

\section{- Pacto Mundial}

Este instrumento de Responsabilidad Social Empresarial fue objetivo de la reunión convocada en Davos por el Secretario General de las Naciones Unidas, Kofi Annan el 31 de enero de 1999. La invitación se extendió a importantes líderes de la economía mundial, para aconsejar la atención voluntaria en diez principios básicos. En el pacto están resumidos la Declaración Universal de los Derechos Humanos, los Principios de la OIT y los estándares de comportamiento con el medio ambiente, que se encuentran fundamentados en las Declaraciones de Copenhague y de Río.

Este pacto de Responsabilidad Social es un acuerdo entre la Organización de Naciones Unidas directamente y las empresas. ${ }^{6}$ Los miembros del acuerdo deben implementar el pacto con los diez principios en "Buenas Prácticas" y participar además en los diálogos políticos, que la misma organización promueve.

El pacto se pone en entredicho (Köpke y Röhr, 2003; Brühl et. al. 2004), especialmente por la diferencia de intereses que persiguen las partes, puede ocurrir que las instituciones miembro se apropien de los fines y objetivos de la contraparte y los acojan como propios. Por ejemplo, puede ocurrir que las empresas ignoren los estándares laborales y del medio ambiente para cumplir con los compromisos y deberes adoptados al comprometerse con la ONU. También surge la pregunta sobre la influencia que puedan tener las organizaciones económicas sobre la ONU, si esto puede representar un ejemplo positivo para la política mundial.

54 Kasten Nowrot se refiere a las redes internacionales que construyen diferentes organizaciones, tanto gubernamentales como no gubernamentales, y otras que sirven a las organizaciones supranacionales como asesores.

6 http://www.unglobalcompact.org 


\section{- Declaración Tripartita de Principios}

La Organización Internacional del Trabajo (OIT), en la que participan todos los Estados, se convierte en una oportunidad de intercambio entre representantes de los empleadores, empleados y los gobiernos del mundo, para crear una iniciativa de responsabilidad social; al involucrar a estos tres sectores e indiscriminadamente varios países adquiere una especial legitimidad para proponer este tipo de instrumentos sobre estándares sociales (Mahari, 1985). Esta organización sugiere los Principios OIT, instrumento que centra su trabajo en cuatro principios: el derecho a la negociación colectiva, la abolición del trabajo forzado, la abolición del trabajo infantil y la prohibición de la discriminación en el trabajo; deben ser automáticamente acogidos por los Estados que han firmando el pacto, por lo tanto, son los mismos Estados los responsables de su cumplimiento.

En 1977 la OIT impulsó la "Declaración Tripartita de Principios sobre las Empresas Multinacionales y la Política Social", 7 instrumento de relación directa respecto a las actividades de las empresas multinacionales, que consiste en un catálogo de reglas sociales, para establecer instrumentos internacionales destinados a reglamentar la conducta de las empresas multinacionales y a fijar las condiciones que han de presidir las relaciones de estas empresas en los países huéspedes, sobre todo en los países en desarrollo. Las relaciones laborales deben fundamentarse en los reglamentos jurídicos nacionales del trabajo.

Dentro de esta iniciativa se tiene en cuenta, al igual que en los instrumentos anteriores, la Declaración Universal de los Derechos Humanos, y otros convenios internacionales sobre política social. Este catálogo no es jurídicamente obligatorio, pero se puede exigir a las empresas que han hecho esta opción, hacer públicas las acciones que han seguido para implementar la Declaración Tripartita.

Los principios de la declaración ofrecen una base para la creación de los Códigos de Conducta que, entre otros aspectos, desean apoyar y fomentar el aumento de la ocupación que ofrecen las empresas, el derecho fundamental de la no discriminación, la seguridad en el trabajo, educación y capacitación para el desarrollo humano y, con esto promover el pago de salarios dignos, óptimas condiciones del sitio de trabajo y el derecho a organizarse libremente y a la negociación colectiva.

\section{- $\quad$ Directrices - OECD}

La Organización para la Cooperación y el Desarrollo Económico (OECD) compuesta por 30 países miembros, tiene como objetivo planear y coordinar las actividades de cooperación y desarrollo de todo el mundo se dedica a formular proyectos de investigación para pronosticar el Desarrollo Económico y también

7 http://www.ilo.org/public/english/employment/multi/download/spanish.pd 
los cambios sociales y tecnológicos de países menos desarrollados especialmente. Igualmente trabaja en un documento en el que sugiere a las empresas adoptar medidas para que la actividad económica no esté en contra con los derechos humanos, este proyecto se llama Directrices de la OECD para Empresas Multinacionales. (OECD, 2000)

La Organización para la Cooperación y el Desarrollo Económico en un intento para crear una guía para las multinacionales, invita a las ONG, representantes de los sindicatos, representantes de los Estados y de las empresas, con el objetivo de ser asesorada. Esta herramienta se crea sin una regulación vinculante y se basa en el principio de la voluntariedad. Se revalúan en esta propuesta los estándares sociales y del medio ambiente y, por lo tanto, los Derechos Humanos. Esta directriz no se limita a cubrir con sus "reglas" a los países OECD sino que se extiende a todas las empresas que tiene relación con países OECD (Hamm, 2005; Brühl. 2004). Los Estados miembro han suscrito este compromiso para promover, asegurar, implementar y respetar la directriz. Aunque la directriz está hecha para ser aplicada directamente por las empresas, ha sido aprobada sólo por un grupo de firmas transnacionales. Incluye importantes disposiciones para las firmas, ellas deben: "Respetar los derechos humanos de todos aquellos afectados por la actividad de la empresa, de acuerdo a los deberes y compromisos del gobierno local y de las obligaciones internacionales."

\section{- Códigos Privados de Empresas}

Con base en la crítica de los grupos de consumidores y, en general, de la opinión pública, las empresas mismas han diseñado y redactado Códigos de Conducta privados, su uso es cada vez mayor y gana aceptación entre los administradores (Köpke y Rohr 2003). Estos códigos de las empresas son declaraciones escritas y voluntarias sobre reglas éticas que tienen como objetivo dirigir o guiar el comportamiento de la empresa y sus actividades en la relación con los grupos interesados que son los accionistas, los clientes, los proveedores, la comunidad y el gobierno. Las Empresas se basan en las legislaciones nacionales y la reglamentación de la Organización Internacional del Trabajo (OIT), para crear sus códigos internos, esto quiere decir que contiene estipulaciones claras sobre, no discriminación laboral, prohibición del trabajo infantil, libertad de asociación, derecho a negociación colectiva e ingresos que aseguren los medios de vida. Existen varios estudios críticos sobre estos códigos de las empresas, se afirma que sus lineamientos son muy cortos y vagamente realizados y se limitan a la empresa, lo que significa que los códigos no son válidos para los proveedores quienes generalmente no tienen ningún tipo de lineamientos (Greven, 2004). 


\section{Las Organizaciones no Gubernamentales (ONG)}

Estas organizaciones escogidas para este trabajo tienen en común su trabajo en defensa de los derechos humanos, han identificado algunas empresas multinacionales trasgresoras de los derechos de los ciudadanos en países como México, Colombia, India e Indonesia, entre otros. Por esta razón han dedicado parte de sus recursos a hacer públicos los casos graves de violación de derechos humanos y la efectividad de la aplicación que hacen las empresas criticadas de los Códigos de Conducta.

Las ONG se pueden identificar por tres características importantes ya que no tienen relación con el Estado o con partidos políticos, entonces no tienen intereses políticos; el trabajo de estas organizaciones se enfoca generalmente a un tercer actor, tienen una función de intermediarias, por lo que relacionan a un financiador con un grupo objetivo, y finalmente, las ONG trabajan sin ánimo de lucro.

Estas organizaciones son activas en la construcción, establecimiento y elaboración de reglas sobre la actuación de las empresas, pues es un reto de estas organizaciones estar del lado de y proteger las personas o grupos sociales vulnerables. Por esto, ellas se convierten en un medio para lograr la armonía en el sistema que, hoy por hoy, se basa en privatizaciones y en disminución de gastos públicos (Wegner, 1993). De igual manera, después de la segunda guerra mundial estas instituciones adquieren un rol importante en la protección de los derechos humanos, estas organizaciones han aumentado considerablemente tanto en número como en tamaño y tienen una gran influencia en el desarrollo internacional (Heintzen, 1988). La cosntrucción de redes globales entre las $\mathrm{ONG}$ es importante para el intercambio de información, y con esto concentrar la atención internacional hacia un punto o espacio específico en el mundo.

Las ONG, desarrolladas con unas ideas propias de crear alternativas de movilización política y participación representan, en muchos casos, un alivio para las obligaciones de los Estados en diferentes áreas en la política social del actual neoliberalismo. (Wegner, 1993) Hoy, estas instituciones trabajan en el área de diseño de leyes, especialmente en la cooperación con organizaciones internacionales y, en algunos casos, donde la democracia es transparente con los Estados (Brühl, Feld, Hamm, Hummel y Martens, 2001).

Para este estudio la posición de cuatro organizaciones que desarrollan sus actividades en Alemania es relevante. Estas organizaciones han ganado un lugar en la política del país y juegan un papel en la toma de decisiones en muchos aspectos nacionales. La característica de estas ONG para ser seleccionadas es el trabajo en el tema de la responsabilidad social y por su ámbito internacional, especialmente porque se refieren a empresas multinacionales que desarrollan actividades económicas de producción en países en vía de desarrollo. 
Las organizaciones tomadas en cuenta son Misereor; Economía, Ecología y Desarrollo Mundial; Amnesty Internacional y Germanwatch. "Misereor" es una ONG que representa el aparato de beneficencia de la iglesia católica en Alemania, es la obra episcopal de cooperación al desarrollo ${ }^{9}$, con actividades en varios países del hemisferio sur, basa su trabajo en el lema ayuda para la auto-ayuda. Economía, Ecología y Desarrollo Mundial, WEED, (World Economy, Ecology and Development) es una organización no gubernamental independiente, se dedica a trabajar en el área de la economía y de la política, su lema es "crear conciencia en Alemania sobre las causas de los problemas de pobreza y medio ambiente" ${ }^{\text {"10 }}$ y "Amnesty Internacional" (ai) es una organización reconocida mundialmente por su trabajo fundamentado en la Declaración Universal de los Derechos Humanos, su misión es "realizar labores de investigación y emprender acciones para impedir y poner fin a los abusos graves contra los derechos civiles, políticos, sociales, culturales y económicos"11. Y, finalmente, "Germanwatch" organización independiente trabaja por la equidad entre el norte y el sur, se ocupa estudiando los efectos mundiales de la economía del norte y de las relaciones norte-sur.

Para Amnistía Internacional las Normas ONU son "un lógico desarrollo y ampliación de los argumentos generales sobre la Declaración Universal de los Derechos Humanos en comparación con las otras alternativas. Las normas son complementarias y ayudan a la implementación de otros estándares existentes" (ai, 2004). Las Normas son una lista de estándares sobre derechos humanos que se debe usar como punto de partida para el desarrollo de la actividad empresarial, de tal forma que la actividad económica de las empresas esté enmarcada con estándares internacionales. Por lo tanto, las Normas ONU y sus comentarios deben tener marcos jurídicos para las empresas, que sean reconocidos a nivel internacional y los gobiernos deben hacer que estas normas jurídicas se reflejen en sus legislaciones nacionales. Las normas igualmente deben alentar las buenas acciones, así como motivar una nueva conformación jurídica de las empresas. Con las normas como referencia se podrá entablar un diálogo entre las empresas y las organizaciones gubernamentales, pueden ayudar para simplificar la toma de decisiones de las empresas.

Según Germanwacht, (en prensa, 2004), ONG tenida en cuenta para esta revisión documental las Normas ONU no son muy mencionadas por la Comisión, la discusión que se hace con respecto a la regulación de la actividad de las empresas, dentro de esta institución es muy limitada y sin perspectivas. Además, se confirma la opinión de otras organizaciones, que afirman que las Normas han encontrado resistencia de parte de las empresas, organizaciones de empresas y los gobiernos de algunos países de Europa y Estados Unidos se resisten contra las Normas (Fonari, 2004), y son la causa de discusiones en la Comisión de Derechos Humanos.

http://www.misereor.org/index.php?L=2\&id=2990

$10 \mathrm{http} / / / \mathrm{www} . w e e d-o n l i n e . o r g /$ about/index.html

11 http://www.es.amnesty.org/quienes-somos/nuestros-objetivos/ 
CorneliaHeydenreich,(enFonari, 2004) encargada de la sección de responsabilidad social de Germanwacht afirma sobre las Normas ONU que presentan procesos de monitoreo, plantea manejar un sistema de quejas y demandas, y un procedimiento arbitral de solución de conflictos. Proporciona la posibilidad de reparación y de indemnización para las víctimas. Cuando no se pueda llegar a un acuerdo entre las partes serán las cortes nacionales, las encargadas de tomar decisiones. Especialmente en lo referente a la vigilancia y las posibilidades de sancionar serán necesarias futuras discusiones. Sólo queda la duda sobre la capacidad que tienen las cortes o estamentos judiciales nacionales para la implementación de las normas.

Estas Normas han sido redactadas de forma tal, que son dirigidas y tienen influencia sobre las actividades de las empresas multinacionales y no tanto sobre las medianas y pequeñas empresas locales (Fonari, 2004). La influencia sobre estas últimas firmas es indirecta, según la relación que se establece con las cadenas de proveedores y tendrán algún efecto siempre y cuando las multinacionales las tengan en cuenta al crear o adoptar un Código de Conducta.

La opinión de WEED sobre las Normas ONU, es que el proceso de construcción de este instrumento demuestra que pueden, algún día llegar a ser el cimiento de un pacto del Derecho Internacional como lo fue en su momento la Declaración Universal de los Derechos Humanos (WEDD, 2006). Con un pacto o tratado internacional se puede comprometer a las empresas a fundamentar sus actividades, con estándares de responsabilidad hacia los derechos humanos.

Para Heidi Feldt de WEED (Ceyssens, Feldt y Hörtreiter, 2005), las normas tienen como característica importante, que la destinación de la reglamentación es independiente al origen de las empresas o del lugar donde está su casa matriz o del lugar donde las firmas desarrollan sus actividades, lo que afirmaría, en caso de convertirse en una parte del derecho internacional, que este sistema no es de los Estados, sino que tiene la capacidad de adaptarse o ajustarse para responder a las necesidades que los cambios jurídico-políticos internacionales pueden manejarse con técnicas flexibles de regulación y de control.

Los conceptos expresados por Amnistía Internacional [ai] (2004) sobre el Pacto Mundial, se pueden sintetizar así: Los principios que el pacto propone sean tenidos en cuenta por las empresas, tanto públicas como privadas, se catalogan como muy generales pues ofrecen una guía mínima respecto a los derechos humanos. Las empresas que toman parte de esta iniciativa deben realizar un documento en el cual apoyan los principios. En este documento también exponen de forma pública sus actividades. El pacto no cuenta con un mecanismo de control. No existe un criterio para determinar qué empresa puede hacer parte del convenio, por esto se afirma que las empresas pueden utilizar esta participación como un medio publicitario y crear con esto una imagen de las actividades de la empresa. Para [ai] este instrumento no ha sido hasta el momento efectivo, pues hay incertidumbre para convertir los principios en buenas prácticas. 
La sociedad civil no es tenida en cuenta como posible mecanismo de control. La falta de mecanismos de sanción, en caso que las empresas no cumplan con lo mencionado en los principios, no es un tema. Además se critica la informalidad con que son tratadas las relaciones de la ONU con la sociedad empresarial.

Este instrumento puede seguramente aportar en el tema de la RSE, y sobre todo aproximarse a las actividades de las empresas en países del hemisferio sur. Para Heydenreich la alta participación de muchas empresas en el Pacto Mundial no es un indicador del compromiso de las empresas con el respeto a los Derechos Humanos. Cada vez más se ve al pacto como un instrumento que sirve de argumento para oponerse a otras medidas y regulaciones (en prensa, 2004) ${ }^{12}$.

Además Germanwacht (en Fonari, 2004) define al Pacto Mundial como "una formulación muy general, no tiene en cuenta la actividad de las empresas proveedoras, no hay un monitoreo para conocer si los principios se cumplen. Los informes no se controlan, no existe un mecanismo de reclamos, ni de sanciones". Y afirma que las tareas de las empresas que participan en el Pacto Mundial, son cada vez menos y sus actividades menos transparentes. Inicialmente las empresas debían preparar un informe anual sobre la aplicación de los principios del pacto, después se hizo obligatorio presentar un ejemplo al año sobre "Buenas Prácticas". Ahora las empresas sólo deben comunicar en el informe final sobre sus actividades para implementar el Pacto Mundial.

Para la señora Strohscheidt, encargada del área de RSE de Misereor (Bussler y Fonari, 2005), los más grandes violadores de los derechos humanos serán muy difíciles de detectar por medio de las actividades que demanda el Pacto Mundial. El pacto solicita informes anuales en los cuales se detallen las "buenas prácticas" de las empresas como mecanismo de ejecución de RSE, pero esta actividad despierta incertidumbre, respecto al verdadero desempeño de las empresas. Las actividades demarcadas dentro de "buenas prácticas" no tienen criterios para ser diferenciadas de las "prácticas normales" de la empresa. Estas pueden ser generalizadas y pueden servir de ejemplo para otras empresas.

Sobre la Declaración Tripartita de la OIT las opiniones de las ONG son breves, se afirma, de manera general, que las recomendaciones contenidas en los convenios son únicamente obligatorias para los Estados miembros que han ratificado los tratados. Es una obligación para los gobiernos hacer que las organizaciones, los empresarios y otras instituciones que se acojan voluntariamente a los principios propuestos. La OIT juega un papel importante para los Estados miembro en cuanto a la asesoría que puede prestar a los gobiernos sobre mecanismos de control y cómo desarrollar procesos, cuando se trate de demandas. Existen algunos principios que son únicamente de carácter voluntario, que se convierten en consejos relacionados

http://www.germanwatch.org/zeitung/2004-1-Global Compactom.htm 
a las solicitudes de los empleados como educación y cursos de perfeccionamiento, condiciones de trabajo y otras relaciones laborales.

La Directriz - OECD según las organizaciones es extensa jurídicamente y hace referencia a un tratado internacional. Es interesante incluso para las ONG y los países en vía de desarrollo ya que posee herramientas prácticas para la denuncia de abusos además de tener amplia cobertura internacional. Son los gobiernos, hasta cierto punto, quienes tienen la responsabilidad del cumplimiento de la directriz, lo que la hace atractiva por poseer legitimidad gubernamental, con el inconveniente de la corrupción que existe en algunos países, lo que impide una óptima práctica de los derechos que se quieren fortalecer por medio de la directriz. Se proyecta dentro de la directriz, la creación de una instancia gubernamental en algunos países, en la que es posible alertar y denunciar; estos espacios son llamados Puntos Nacionales de Contacto (PNC). Cualquier persona que se considere afectada por la actividad de la empresa tiene la posibilidad de denunciar ante los PNC del país donde la infracción fue cometida o en el país de origen de la empresa, donde está ubicada la casa matriz.

Las mayores críticas (Kerkow, Martens y Schmitt. 2003; Bussler y Fonari. 2005) sobre la directriz se basan en la voluntariedad del instrumento. Además de su validez sólo para empresas de países OECD y ubicados en países OECD, la cobertura de la directriz para las empresas proveedoras es limitada. No se tratan temas sobre salarios que aseguren los medios de vida y el trabajo en horas extras. Los fallos de los casos admitidos en el PNC, pueden requerir de un largo periodo de tiempo, asimismo estos casos pueden pasar a ser negociaciones jurídicas de carácter nacional, lo que implica complicadas negociaciones burocráticas, en algunos casos. Si ocurre incumplimiento por parte de una empresa que se niega a reparar los daños, queda sólo el criterio público para hacer demandas.

Las ONG (WEED et. al., 2006) concuerdan en varios puntos con respecto a su opinión sobre los Códigos de Conducta Privados, de forma general: la crítica más relevante de este instrumento se concentra en los mecanismos de control y las formas de sancionar ante la trasgresión de los códigos. Cuando la aplicación y el cumplimiento de estos reglamentos no son llevados a cabo por un organismo independiente, existe la posibilidad que las firmas abusan de los códigos. También se afirma que los criterios seleccionados por las empresas mismas no son suficientes para introducir reglas sociales y ecológicas. Las empresas observan la presión masiva y los escándalos para poner en práctica grandes campañas, construyen Códigos de Conducta como alivio para evitar reacciones de desprestigio de los consumidores, sin que se garanticen hechos concretos y seguimiento de los compromisos que ellos mismos han asumido. Las firmas necesitan mantener una imagen positiva ante el público, precisamente en los países donde las empresas tienen sus casas matrices son puntos claves para mostrar sus medidas de orden jurídico. Para las empresas las obligaciones internas voluntarias son herramientas óptimas para disminuir las reglamentaciones externas y así disminuir el costo burocrático, estas reglamentaciones propias pueden ayudar a 
mantener la reputación; las empresas logran una buena imagen, al demostrar que son responsables y mostrarse sensibles.

Para Germanwacht es claro que no solamente es cuestión de las empresas fomentar medidas de protección de los derechos humanos y controlar que se cumplan, también depende de las acciones y reglamentaciones de los Estados. Estos códigos deben servir como complemento para las normas legales, deben poseer un carácter jurídicamente vinculante.

Cada vez más y debido a la presión pública, las empresas introducen conceptos de Responsabilidad Social en los cuales los Códigos de Conducta Internos son importantes. Estos códigos varían con respecto al alcance, contenido, práctica y control. "Existe una gran tendencia del uso de estos instrumentos, es reconocida para fundamentar una misión o visión empresarial, pero explícitamente la relación con los derechos humanos es muy vaga" (Fonari, 2004 p.66).

“Los códigos son también sólo compromisos de boca de las empresas y son usados para recuperar la imagen que constantemente pierden las firmas por sus actitudes negligentes hacia sus trabajadores y el medio ambiente" (Bussler y Fonari, 2005).

Para WEED es un punto de análisis para la formulación y aplicación de estos compromisos voluntarios el tener en cuenta la opinión de las uniones de trabajadores, las ONG y otros grupos internos y externos de la empresa, este es un aspecto de la seriedad de los códigos privados. Por lo tanto, "esos compromisos voluntarios se pueden ver como un escalón de medio corazón ${ }^{13}$ en la dirección correcta". ( Kerkow, Martens y Schmitt, 2003, p.5)

\section{Conclusión}

Cada vez más, existe un reconocimiento, de la importancia de aumentar el respeto y la promoción de los derechos humanos, lo cual se ve reflejado en todas estas nuevas propuestas tanto privadas como públicas $\mathrm{y}$, en el número de personas involucradas en el tema en todos los países del mundo. Una empresa de hoy no sólo es competitiva si demuestra beneficios económicos, es indispensable introducir conceptos sociales en su ámbito interno y externo; es el caso la RSE que tiene como objetivo final contribuir positivamente a la sociedad, a través de la planeación de proyectos en los que se construyen e implementan Códigos de Conducta.

Los proyectos que desarrollan las empresas sobre responsabilidad social constituyen un buen punto de partida para mejorar las relaciones de estas con el medio ambiente y con las personas a quienes influyen con su actividad económica. Para poder lograr los objetivos propuestos, se requiere entonces de un marco

13 Traducción textual de la expresión alemana: Halbherzig 
normativo que regule la actividad de las empresas de forma global, pero también la transformación de las empresas de forma interna. Este proceso será efectivo siempre y cuando el trabajo sea conjunto.

Las empresas no quieren ser relacionadas con temas de violación de los Derechos Humanos, pues temen a campañas de desprestigio. Con el objetivo de evitar las denuncias públicas las empresas recurren a construir campañas de RSE y utilizar como instrumentos los Códigos de Conducta que son una forma práctica y sencilla de presentar sus buenas prácticas dentro de su actividad económica.

Las ONG han adquirido en los últimos años un papel importante en temas sociales y ambientales. Han hecho énfasis sobre la discrepancia entre el discurso y la acción de algunas empresas, han adoptado una función de "watchdog" (perro que cuida), han representando los intereses de víctimas, estas actividades han posicionado a las ONG ante empresas multinacionales como organismos de control, también se han logrado importante avances, han cambiado las relaciones y las políticas de muchas instituciones, especialmente en temas ambientales. Otra práctica importante de las ONG es hacer campañas publicitarias abiertas que denuncien las violaciones de los Derechos Humanos, que tienen como objetivo, sensibilizar a los consumidores e influir en la adquisición o en restringir el consumo de determinados bienes y servicios.

Las empresas que introducen instrumentos de RSE dentro de sus políticas pueden sobrellevar una etapa coyuntural, pero también tienen la posibilidad de encontrar otro camino de competitividad internacional. Este tipo de prácticas puede ser usado según los intereses y la posición del público, los Códigos de Conducta son una herramienta que ofrece diversas posibilidades para guiar programas de RSE que se pueden enfocar de acuerdo a las necesidades de la empresa. El carácter voluntario de la mayoría de instrumentos es una ventaja para las empresas; que les permite diseñar el proceso que desean seguir. Las empresas que desarrollen estos procesos seguramente podrán contar con posibilidades ventajosas en el mercado y demostrar un comportamiento responsable.

Los Códigos de Conducta son intentos de las instituciones en el área de la RSE que representan los primeros pasos para la solución de problemas en muchos espacios. Estos códigos pueden regular las operaciones transnacionales de un número creciente de empresas que aprovechan las ventajas de la globalización. Para las naciones son una herramienta apropiada que sirve para complementar sus reglamentaciones, sus sistemas jurídicos y de control, en aspectos que se relacionan con la actividad internacional de las empresas.

Los códigos de conducta, son buenas iniciativas e instrumentos que tienen como fin mejorar condiciones sociales, pero ellos no pueden sustituir ninguna legislación internacional o nacional, tampoco en lo concerniente a lo ambiental, social, ni laboral, el respeto de las normas es cuestión de hacerlas válidas, legítimas y según 
las ONG, sancionables. Los códigos pueden ser herramienta que los trabajadores y sus representantes $y$, en general, la sociedad civil puede utilizar para lograr que se respeten los derechos humanos en varios ámbitos, especialmente en las relaciones laborales, ambientales y sociales de las empresas nacionales y multinacionales.

\section{Referencias}

Alta comisión de Derechos Humanos. (2005). Human rights and transnational corporations and other business enterprises., Human Rights Resolution 2005/69 E/CN.4/2005/L.10/Add.17

Amnesty International, Decisión 2004/116. (2004). "Responsibilities of Transnational corporations and related business enterprises with regard to Human Rights.

Bass, H. H., \& Melchers, S. (2004). Neue Instrumente zur sozialen und ökologischen Gestaltung der Globalisierung: Codes of Conduct, Sozialklauseln, nachhaltige Investmentfonds. Münster: Lit-Verl

Boyle, Alan. (1999), International Law and Sustainable Development: Past Achievements and Future Challenges, Editor David Freestone

Brigitte, H. (kein Datum). Universidad de Munster. Von Recht auf Entwicklung Seine Bedeutung für die Zukunft: http://www.uni-muenster.de/PeaCon/wuf/wf96/9630210m.htm abgerufen

Brühl, T., Feld, H., Hamm, B., \& Hummel, H. (2004). Unternehmen in der Weltpolitik. Politiknetzwerke, Unternehmensregeln und die Zukunft des Multilateralismus. Bonn: Verlag J.H.W. Dietz Hachfolger

Bussler, C., \& Fonari, A. (2005). Sozial- und Umweltstandards bei Unternehmen. Chancen und Risiken. Münich: Eine Welt Netzwerk Bayern e.V.

Ceyssens, J., Feldt, H., \& Hörtreiter, I. (2005). Zwischenstaatliche Instrumente zur Stärkung der Unternehmensverantwortlichkeit. Berlin: WEED

Comisión de Derechos Humanos, Sub-Commission on the Promotion, and Protection of Human Rights. (2005). Norms on the responsibilities of transnational corporations and other business enterprises with regard to human right. UN

Comisión de la Unión Europea. (2002). Mitteilung der Kommission: Die soziale Verantwortung der Unternehmen: ein Unternehmensbeitrag zur nachhaltigen Entwicklung. Brüssel: $\operatorname{KOM(2002)~} 347$

Cortés, G., Eichler, B., Rau, J., \& Wieczorek-Zeul, H. (2004). Arbeitnehmerrechte in einer Globalisierten Welt. Hamburgo: VSA Verlag

Faden, M. (2002). Bedeutung und Rolle der menschenrechte im Rahmen der Welthandelsorganisation (WTO). Hamburg: HWP. 
Florence, G. (29. 03 2004). Konzerne müssen die Menschenrechte respektieren! - Die Erklärung von Bern. EVB Suiza

Fonari, A. (2004). Menschenrechts-, Arbeits- und Umweltstandards bei multinationalen Unternehmen. Münich: Germanwatch Regionalgruppe Münchner Raum \& Europäische Akademie Bayern e.V

Friedman, M. (1971). Kapitalismus und Freiheit. Stuttgart: Seewald Verlag.

Friedmann, M. (13. 09 1970). The Social Responsibility of Business is to Increase its Profits. The New York Times Magazine

Fryas, J. G., \& Pegg, S. (2003). An Emerging Market for the new Millennium: Transnational Coorporations and Human Rights in Transnational Corporations and Human Rights. New York: Pelgrave MacMillan

Germanwatch. (8 2005). Publicaciones y Declaraciones Germanwacht. Von http:// www.germanwatch.org/zeitung/ abgerufen

Germanwatch. (25. 02 2004). Unverbindlich bei verbindlichen Regeln für Unternehmen, Kommentar von Germanwatch zum Bericht der ILOWeltkommission. Germanwatch Pressemitteilung

Greven, T. (2004). Private, Staatliche und überstaatliche Intervention zur Verankerung von Arbeitnehmerrechten”. Bonn: Bass, $\mathrm{H}$

Greven, T., \& Scherre, C. (2005). Globalisierung gestalten, Weltökonomie und soziale Standards. Bonn: Bundeszentrale für Politische Bildung

Hamm, B. (2004). Maßnahmen zur Stärkung von Corporate Social Responsibility in der Entwicklungszusammenarbeit europäischer Institutionen und ausgewählter europäischer Geberländer. Bonn: Institut für Entwicklung und Frieden (INEF)

Heydenreich, C. (2004). UN-Normen für Unternehmen unterstützen. Bündnis deutscher ONGs bekräftigt Forderungen anlässlich der laufenden Tagung der UN-Menschenrechtskommission. Conferencia de Derechos Humanos. Berlin: Menschenrechtkommission

Kerkow, U., Martens, J., \& Schmitt, T. (2003 ). Die grenzen der Freiwilligkeit, Handlungsmöglichkeiten und Erfahrungen von NGOs und Gewerkschaften bei der Anwendung freiwilliger Selbstverpflichtungen der Wirtschaft. Bonn/Berlin: WEED Arbeitspapier

Köpke, R., \& Röhr, W. (2003). Codes of Conduct, Verhaltensnormen für Unternehmen und ihre Überwachung. Köln : PapyRossa Verlag

Lozano, J. F., \& Siurana, J. C. (2000). Las comisiones éticas como mecanismo de integración de la ética en las organizaciones. Papeles de Ética, Economía y Dirección 
Mahari,J.(1985). "Codes of Conductfürmultinationale Unternehmenunter besonderer Berücksichtigung betriebswirtschaftlicher und juristischer Gesichtspunkte”. Diss. St. Gallen, Hochschule für Wirtschaft- und Sozialwissenschaften. Zurich: Schulthess Verlag

Nowrot, K. ( 2003). Die UN-Norms on the Responsibility of Transnational Corporations and Other Business Enterprises with Regard to Human Rights Gelungener Beitrag zur transnationalen Rechtsverwirklichung oder das Ende des Global Compact? Halle: Tietjie C. \& Kraft G. Beiträge zum Transnationale Wirtschaftrecht. Martin Luther-Universität Halle-Wittenberger

OECD, Organisation Für Wirtschaftliche Zusammenarbeit Und Entwicklung. (2000). Die OECD-Leitsätze für multinationale Unternehmen Neufassung. OECD

Oficina de la alta comisión de Derechos humanosde la Organización de las Naciones Unidas. (12 2005). ONU. Von http://www.ohchr.org abgerufen

Organisation Für Wirtschaftliche Zusammenarbeit Und Entwicklung OECD. (2000). Die OECD-Leitsätze für multinationale Unternehmen Neufassung

Paech, N. (2003 ). Die soziale, ökonomischen und kulturellen Menschenrechte im Rechtsystem der Internationale Wirtschafts- und Handelsordnung. Hamburg: Friedrich-Ebert-Stiftung

Sarasin, E. (2000. 11 11). Simple Gewinnmaximierung ist vorbei. Human Rights Tages-Anzeiger, S. 28

Steinmann, H., Löhr, A., \& Suzuki, S. (2003). Unternehmensethik - 100 Jahre Betriebswirtschaftslehre in Deutschland

Steinmann, H., Löhr, A., \& Suzuki, S. (2003). Unternehmensethik - 100 Jahre Betriebswirtschaftslehre in Deutschland

Strutynski, P., \& Platiel, N. (2005/2004). Artemis am Congo Was hatte die europäische Jagdgöttin in Afrika zu suchen? Uni Kassel, AG Friedensforschung, Informationsstelle Militarisierung (IMI) e.V

WEED, terre des hommes, Global Policy Forum und DGB Bildungswerk. (2006). Verbindliche Regeln für Multis - Corporate Accountability. Zwischenbilanz und Perspektiven. Düsseldorf: WEED, terre des hommes, Global Policy Forum und DGB Bildungswerk

WEED Weltwirtschaft, Ökologie und Entwicklung . (kein Datum). Ein Internationales Investitionsabkommen im Rahmen der WTO? Positionen und Hintergründe aus entwicklungspolitischer Sicht, Mustervortrag. Von http://www2.weed-online. org/uploads/Mustervortrag-Inv abgerufen 
Instrumentos de responsabilidad social empresarial ...

Wegner, R. (1993). Nicht-Regierungsorganisationen als entwicklungspolitische Hoffnungsträger? Eine Studie zur Wirksamkeit privater Entwicklungshilfe auf den Philippinen. Lit Verlag : Hamburgo

Weltwirtschaft, Ökologie \& Entwicklung. WEED. (11 2005). Publicaciones y Declaraciones Weltwirtschaft, Ökologie \& Entwicklung. WEED. Von http:// www2.weed-online.org/uploads/Pr\%FCfsteine_korr1.pdf abgerufen 